\title{
Improving membrane performance by incorporating polymer-embedded metal-organic framework microspheres
}

\author{
Wufeng $\mathrm{Wu}^{1}$, Pengcheng $\mathrm{Su}^{1}$, and Wanbin $\mathrm{Li}^{2}$ \\ ${ }^{1}$ Jinan University \\ ${ }^{2}$ Affiliation not available
}

June 5, 2020

\begin{abstract}
The controlling nanofiller aggregation and strengthening interfacial interaction are of great scientific significance for mixed matrix membranes (MMMs). In this study, the polymer-embedded metal-organic framework (pMOF) microspheres (MSs) are designed by one-pot synthesis and employed as microfillers for improving separation performance of MMMs. Through adding polymer during solvothermal crystallization, the polymer chains are embedded into the MOF materials, and the morphologies of the MOFs are transformed from nanopaticles to polycrystalline MSs. Since the embedding of the identical polymer promotes the compatibility of polymeric matrixes and fillers, as well as the micrometer-sized porous MSs offer additionally superior and permanent transport pathways, the resulted MMMs display simultaneously enhanced selectivity and permeability for carbon capture. The $\mathrm{CO} 2 / \mathrm{CH} 4$ selectivity and $\mathrm{CO} 2$ permeability of the pMOF MMMs are achieved at 1.3 and 2.2 times as those of the pure polymeric membranes, and 1.5 and 1.2 times as those of the MOF MMMs, respectively.
\end{abstract}

Improving membrane performance by incorporating polymer-embedded metal-organic framework microspheres

Wufeng $\mathrm{Wu},{ }^{+}$Pengcheng $\mathrm{Su},{ }^{+}$Wanbin $\mathrm{Li}^{*}$

Guangdong Key Laboratory of Environmental Pollution and Health, School of Environment, Jinan University, Guangzhou 511443, P.R. China

${ }^{+}$W.W. and P.S. contributed equally to the paper.

*Corresponding author. E-mail address: gandeylin@126.com (W. L.)

Abstract: The controlling filler aggregation and strengthening interfacial interaction are of great scientific significance for mixed matrix membranes (MMMs). In this study, the polymer-embedded metal-organic framework (pMOF) microspheres (MSs) are designed by one-pot synthesis and employed as microfillers for improving separation performance of MMMs. Through adding polymer during solvothermal crystallization, the polymer chains are embedded into the MOF materials, and the morphologies of the MOFs are transformed from nanopaticles to polycrystalline MSs. Since the embedding of the identical polymer promotes the compatibility of polymeric matrixes and fillers, as well as the micrometer-sized porous MSs offer additionally superior and permanent transport pathways, the resulted MMMs display simultaneously enhanced selectivity and permeability for carbon capture. The $\mathrm{CO}_{2} / \mathrm{CH}_{4}$ selectivity and $\mathrm{CO}_{2}$ permeability of the pMOF MMMs are achieved at 1.3 and 2.2 times as those of the pure polymeric membranes, and 1.5 and 1.2 times as those of the MOF MMMs, respectively.

Keywords: metal-organic frameworks, polymer-embedding, interfacial compatibility, mixed matrix membranes, carbon capture 


\section{INTRODUCTION}

Excessive emission of carbon dioxide causes severe environmental issues, e.g. global warming. ${ }^{1,2}$ Carbon capture, realized by separating $\mathrm{CO}_{2}$ from other gases, e.g. $\mathrm{H}_{2}, \mathrm{~N}_{2}$, and $\mathrm{CH}_{4}$, has been considered as a feasible strategy to reduce $\mathrm{CO}_{2}$ emission. Various technologies, including absorption and membrane separation, are proposed for $\mathrm{CO}_{2}$ capture. ${ }^{3-8}$ Membrane technology has been attracting intensive attention because of its high efficiency, simple operation, environmental friendliness, etc. ${ }^{7-11}$ Polymeric membranes with high processibility, low energy requirement, and relatively cheap production cost are widely applied in $\mathrm{CO}_{2}$ separation. However, the well-known trade-off between selectivity and permeability hampers the further development of those membranes. ${ }^{12,13}$ Mixed matrix membranes (MMMs) are formed by continuous phase of polymeric matrixes and dispersed phase of particles. ${ }^{14}$ The incorporation of porous particles not only offers additional transport channels but also adjusts free volumes by changing polymer arrangements, thereby leading to the improvement of permeability and selectivity. ${ }^{14,15}$

Many kinds of materials have been applied as fillers to prepare MMMs, e.g. graphene oxide, porous carbons, and metal organic framework (MOFs). ${ }^{16-19}$ Benefiting from the large surface areas, unique adsorption features, and tailorable apertures, MOF materials are commendable candidates for fabrication of membranes with excellent separation performance. ${ }^{20-28}$ Gascon's group substantially improved the capture performance of polyimide membranes by introducing CuBDC nanosheets. ${ }^{29}$ Jiang et al.prepared the ZIF- 8 hollow nanoparticles based MMMs for efficient $\mathrm{CO}_{2}$ capture. ${ }^{30}$ An ideal MMM with desirable property generally consists of uniformly dispersed fillers and excellent polymer/filler compatibility. The MOF sizes have great influence on the microstructures of MMMs. By reason of the large filler/matrix interfacial areas, nano-sized MOF particles are preferred for preparing MMMs. ${ }^{31-33}$ Japip et al.demonstrated that the MMMs incorporated with 200-nm ZIF-71 nanoparticles (NPs) exhibited better selectivity than that contained 600-nm fillers. ${ }^{34}$ However, the excessive surface energy of small NPs may result in filler aggregation. Comparatively, incorporation of large microfillers encounters lower risk of aggregation. Furthermore, the microparticles in MMMs can provide more consecutive transport pathways for gas permeation than nanofillers. ${ }^{35,36}$

Although the great progresses have been achieved in preparation of MOF-based MMMs, the enhancement of MOF/polymer interactions is still ungently desired for better separation. The weaker binding forces between MOFs and matrixes are more likely to cause the invalid defects, which provide the non-selective pathways and deteriorate the selectivity of MMMs. ${ }^{37}$ This phenomenon will be further aggravated for the MMMs with large fillers. In order to finely control the interfacial defects, proper modification of MOF fillers is exploited as the key step for ameliorating filler dispersion and remolding interfacial combination. ${ }^{38}$ The modification is performed through coating or grafting by additional appropriate molecules on MOF surface or at unsaturated metal centers and functional linkers. ${ }^{39-43}$ Jin et al. incorporated polydopamine-modified ZIF-8 into polyimide (PI) to prepare the MMMs with excellent dispersion and compatibility. ${ }^{39}$ Shojaeiet al. grafted $\mathrm{NH}_{2}$-UiO-66 NPs by poly methyl methacrylate (PMMA) to reinforce the interfacial affinity of MMMs. ${ }^{41} \mathrm{Li}$ et al. utilized the chelating effect between the metal nodes of MOFs and the ester groups of crosslinked polyethylene oxide (XLPEO) to strengthen the interfacial interactions of MMMs. ${ }^{42}$ Although chemical modification can ameliorate the dispersion of NPs and improve the interfacial affinity of MMMs, the complicated modification procedures, special requirements to active sites, and risks of blocking MOF pores limit the extensive application.

The large fillers with good compatibility to matrixes, obtained by simple, effective, and versatile synthesis, are highly needed for simultaneously enhancing the permeability and selectivity of MMMs. Herein, we reported employing polymer-embedded MOF (pMOF) microspheres (MSs) as fillers, which were fabricated by one-pot synthesis, to improve the separation performance of MMMs (Figure 1). By adding polymer during crystallization, the polymer chains could be embedded into the MOF materials and could change the MOF NPs to MSs. Because the same polymer embedding as matrix promoted the compatibility of membranes, the prepared MMMs with pMOF fillers displayed improved selectivity for $\mathrm{CO}_{2}$ capture. Meanwhile, since the micrometer-sized MSs offered highly efficient gas transport channels, the permeability of the MMMs greatly increased. 


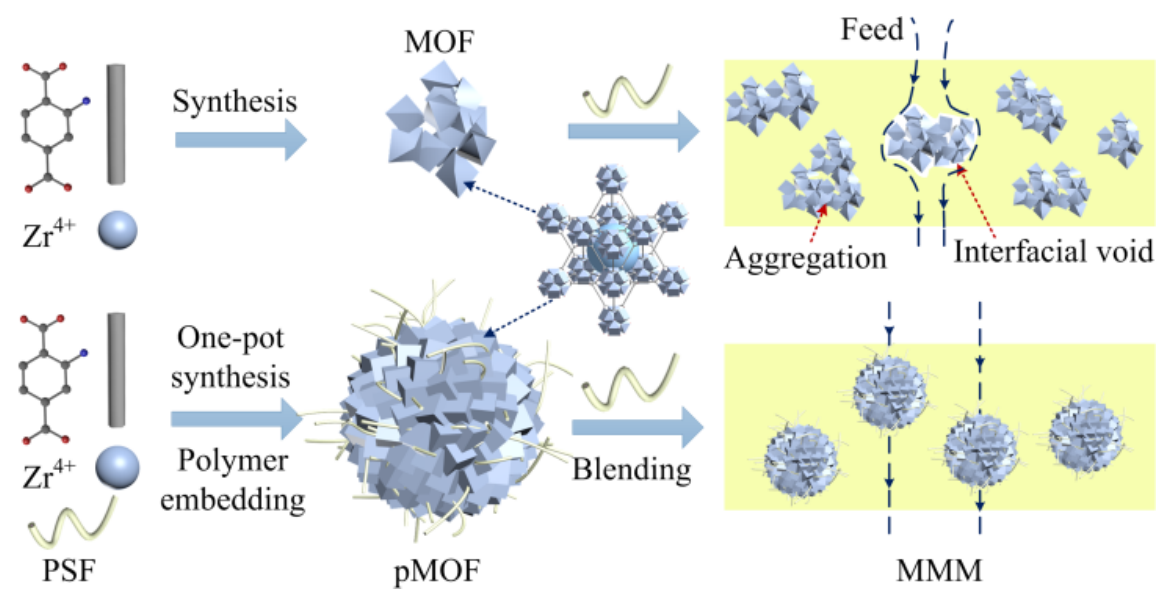

Figure 1. Synthesis procedures for MOF NPs and pMOF MSs along with the formation of MMMs.

\section{EXPERIMENTAL SECTION}

\section{Preparation of $\mathrm{NH}_{2}$-UiO-66 NPs}

$\mathrm{ZrCl}_{4}(0.48 \mathrm{~g})$, 2-aminoterephthalic acid $\left(\mathrm{NH}_{2}\right.$-BDC, $\left.0.37 \mathrm{~g}\right)$, and deionized water $(11.9 \mu \mathrm{L})$ were added in N,N-dimethylformamide (DMF, $40.0 \mathrm{~mL}$ ) and dissolved by stirring and ultrasonic treatment. ${ }^{44}$ The transparent precursor was transferred into a Teflon-lined stainless-steel autoclave. For crystallization, the autoclave was thermally treated at $120{ }^{\circ} \mathrm{C}$ for $24 \mathrm{~h}$. After natural cooling to room temperature, the formed powders were isolated by centrifugation at $6000 \mathrm{rpm}$ for $6 \mathrm{~min}$. Eventually, the obtained powders were washed by DMF and methanol for several times, and dried in vacuum at $100{ }^{\circ} \mathrm{C}$ overnight.

\section{Preparation of polymer-embedded $\mathrm{NH}_{2}-\mathrm{UiO}-66 \mathrm{MSs}$.}

$\mathrm{ZrCl}_{4}(0.48 \mathrm{~g}), \mathrm{NH}_{2}$-BDC $(0.37 \mathrm{~g})$, and polysulfone (PSF, $\left.4.80 \mathrm{~g}\right)$ were dissolved in DMF (80 mL). For dissolving, the suspension was stirred for $4 \mathrm{~h}$. The obtained transparent solution was transferred to a Teflonlined stainless steel autoclave. For crystallization, the autoclave was thermally treated at $120{ }^{\circ} \mathrm{C}$ for $24 \mathrm{~h}$. After natural cooling to room temperature, the formed powders were isolated by centrifugation at $6000 \mathrm{rpm}$ for $6 \mathrm{~min}$. Eventually, the obtained powders were washed by DMF and methanol for several times, and dried in vacuum at $100{ }^{\circ} \mathrm{C}$ overnight.

\section{Fabrication of MMMs}

Before membrane fabrication, the PSF powders, MOF NPs, and pMOF MSs were dried in vacuum at 100 ${ }^{\circ} \mathrm{C}$ overnight to remove the adsorbed water. The MOF NPs and pMOF MSs were employed as fillers. As shown in Eqs. (1) and (2), the amount of the solid substances (polymers and fillers) were $10 \mathrm{wt} \%$ of the mixtures, while the fillers accounted for $10 \mathrm{wt} \%$ of the solid substances. The fillers were first dispersed in $\mathrm{CHCl}_{3}$ to prepare the initial suspension, followed by constant stirring and ultrasonic treatment for $2 \mathrm{~h}$. The PSF powders were added into the above suspension and then stirred overnight. To completely remove the air bubbles, the casting ink was ultrasonically treated for $5 \mathrm{~min}$ and stirred for $5 \mathrm{~min}$, repeating this process for 6 times and then standing for $1 \mathrm{~h}$. The prepared viscous membrane ink was poured on a glass plate and casted with a scraper. After standing at room temperature for $1 \mathrm{~h}$, the formed membrane was placed in an oven at $50{ }^{\circ} \mathrm{C}$ for $4 \mathrm{~h}$, and then dried in vacuum at $80{ }^{\circ} \mathrm{C}$ overnight to completely evaporate the residual solvent. The pure PSF membrane was prepared by the same method as above.

$$
\begin{aligned}
& \frac{\mathrm{PSF}+\mathrm{MOF}}{\mathrm{PSF}+M O F+\mathrm{CHCl}_{3}}=10 w t \%(1) \\
& \text { MOF loading }(w t \%)=\frac{\mathrm{MOF}}{\mathrm{PSF}+M O F} \times 100=10 w t \%(2)
\end{aligned}
$$

\section{Separation performance}


The gas transport behaviors of the prepared membranes were analyzed by using a reequipped gas permeation analyzer (MGT-01, Labthink CO.). The prepared membranes with exposed area of $3.14 \mathrm{~cm}^{2}$ were sealed in permeation cell by O-ring. After vacuum treating the permeation system, the feed chamber was filled with the gas to be measured with pressure of $100 \mathrm{kPa}$. The permeate gas was analyzed by gas chromatography. The gas permeability (P, Barrer, 1 Barrer $=10^{-10} \mathrm{~cm}^{3}(\mathrm{STP}) \mathrm{cm} \mathrm{cm}^{-2} \mathrm{~s}^{-1} \mathrm{cmHg}^{-1}$ ) was calculated based on the permeate gas volume, membrane thickness, effective area, permeation time, and transmembrane pressure. The selectivity $\left(\alpha_{i}\right)$ was calculated by the permeability of two gases:

$\alpha_{i}=\frac{P_{\mathrm{CO}_{2}}}{P_{\mathrm{CH}_{4}}}(3)$

\section{Characterizations}

The morphologies of the MOF particles and MMMs were observed by using a field-emission scanning electron microscope (SEM) (Ultra-55, Zeiss Co.). X-ray diffraction (XRD) (D2 Phaser, Bruker CO.) was applied to characterize the crystalline structure of the prepared samples. X-ray photoelectron spectroscopy (XPS, A RBD upgraded PHI-5000C, ESCA system, PerkinElmer) and Fourier transform infrared spectroscopy (FTIR, IRTracer-100, Shimadzu CO.) were employed to study the chemical structure of the prepared samples. $\mathrm{N}_{2}$ adsorption-desorption isotherms was collected by using a physisorption analyzer (Autosorb iQ Station 1, Quantachrome Co.) at $77 \mathrm{~K}$ to obtain the Brunauer-Emmett-Teller (BET) surface area and pore size distribution. The gas sorption properties of the prepared samples were tested by using the physisorption analyzer at $25^{\circ} \mathrm{C}$.

The adsorption selectivity selectivities of the MOF NPs and pMOF MSs were calculated by Henry's Law: ${ }^{25}$

The Henry's laws constants $\mathrm{K}_{H}$ was defined:

$\mathrm{K}_{H}=\frac{\mathrm{dn}}{\mathrm{dp}}(4)$

The Henry's law selectivity for pure gases was obtained as:

$S_{i, j}=\frac{K_{H, i}}{K_{H, j}}(5)$

where $i$ and $j$ were different gases, $K_{H, i}$ and $K_{H, j}$ were Henry's law coefficient.

A dual sorption theory was used to describe sorption isotherm of the MMMs: ${ }^{45,46}$

$C=C_{D}+C_{H}=k_{D} p+\frac{c_{H}^{\prime} \mathrm{bp}}{1+\mathrm{bp}}(6)$

where $C\left(\mathrm{~cm}^{3}(\mathrm{STP}) \mathrm{cm}^{-3}(\mathrm{MMM})\right)$ was solubility. $C_{D}$ and $C_{H}$ represented the amount of gas adsorbed in Henry and Langmuir sites, respectively. $k_{D}\left(\mathrm{~cm}^{3}(\mathrm{STP}) \mathrm{cm}^{-3}(\mathrm{MMM}) \mathrm{kPa}^{-1}\right)$ and $c^{\prime}{ }_{H}\left(\mathrm{~cm}^{3}(\mathrm{STP}) \mathrm{cm}^{-3}(\mathrm{MMM})\right)$ referred to Henry's solubility and Langmuir saturation constants, respectively. $b\left(\mathrm{kPa}^{-1}\right)$ and $p(\mathrm{kPa})$ were the Langmuir affinity constant and pressure, respectively.

The solubility coefficient $(S)$ of gas in membranes was calculated by:

$S=\frac{C}{p}=k_{D}+\frac{c_{H}^{\prime} b}{1+b p}$

The diffusion coefficient $(D)$ was calculated from permeability $(P)$ and $S$ :

$D=\frac{P}{S}(8)$ 

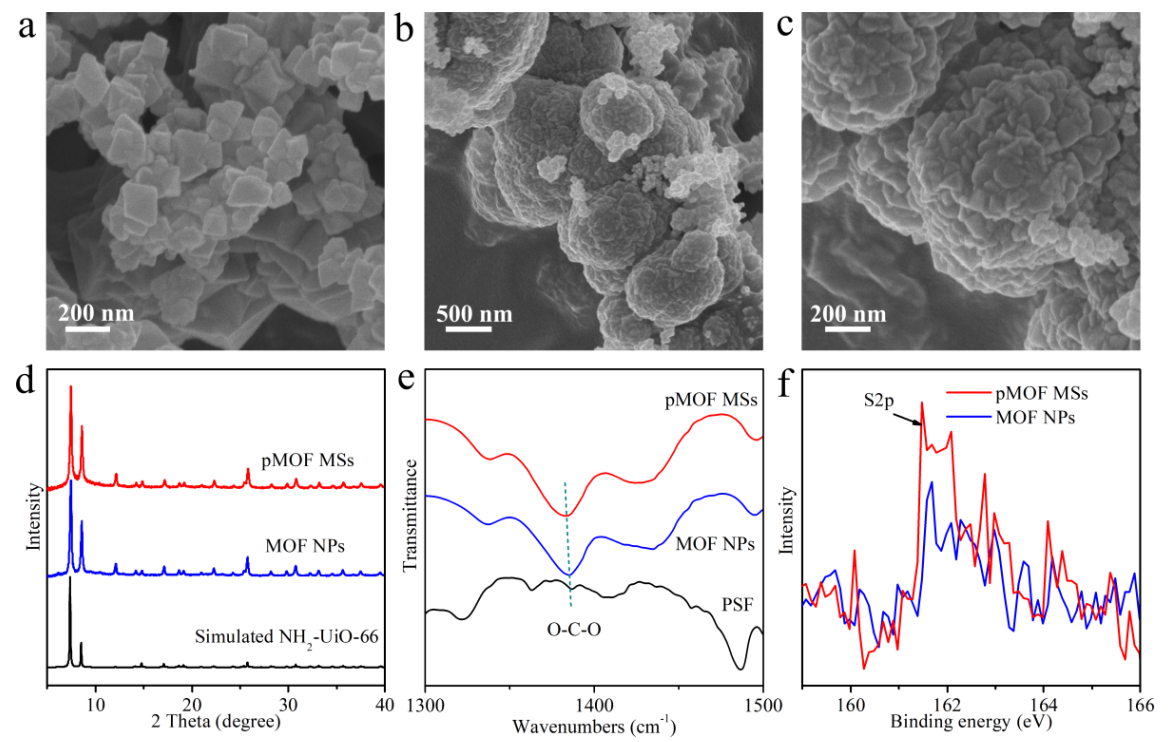

Figure 2. SEM images of the (a) MOF NPs and (b,c) pMOF MSs. (d) XRD patterns of the simulated $\mathrm{NH}_{2}$-UiO-66, MOF NPs, and pMOF MSs. (e) FTIR spectra of the PSF, MOF NPs, and pMOF MSs. (f) High resolution S 2p XPS spectra of the MOF NPs and pMOF MSs.

\section{RESULTS AND DISCUSSION}

\section{Preparation of MOF NPs and pMOF MSs}

To illuminate the concept of improving separation performance of MMMs by incorporating pMOF MSs, we employed $\mathrm{NH}_{2}-\mathrm{UiO}-66$ as filler and PSF as matrix. As one of the most studied MOFs materials, $\mathrm{NH}_{2}-\mathrm{UiO}-$ $66,{ }^{47,48}$ constructed by the coordination between $\mathrm{Zr}_{6} \mathrm{O}_{4}(\mathrm{OH})_{4}$ centers and $\mathrm{NH}_{2}$-BDC linkers, has excellent chemical stability and good adsorption capacity for $\mathrm{CO}_{2} \cdot{ }^{49} \mathrm{PSF}$ with cost-effective and thermally stable features possesses great potential in fabrication of high-performance membranes. ${ }^{50,51}$ Figure 1 showed the synthesis procedures of the MOF NPs and MSs. The $\mathrm{NH}_{2}$-UiO-66 MSs were obtained by the simple one-pot synthesis via introducing PSF in precursor solutions, while the $\mathrm{NH}_{2}-\mathrm{UiO}-66 \mathrm{NPs}$ were prepared without polymer additives. On account of solubility, the polymer was added with concentration of $6.0 \mathrm{wt} / \mathrm{v} \%$ in precursor solutions, because the dissolutions of polymers and precursors influenced each other. As shown in SEM images (Figure 2a), the $\mathrm{NH}_{2}$-UiO-66 NPs without polymer additives possessed well-defined octahedral structure, with the size of 150-200 nm. For the polymer-embedded pMOF MSs, the obvious different spherical structure appeared (Figure 2b,c). The size of pMOF microspheres was about 1-2 $\mu \mathrm{m}$, which was much larger than that of the MOF NPs. During the crystallization process of MOFs, the added polymer chains in precursor solution prompted the assemblage of the nuclei and the intergrowth of crystals, thereby leading to the formation of large polycrystalline spherical structures. ${ }^{52,53}$ Moreover, it was noticeable that the prepared pMOF MSs possessed relatively rough surface, which might be beneficial to improve the interfacial interaction between MSs and matrixes due to the enlarged contacting area.

\section{Characterizations of MOF NPs and pMOF MSs}

The crystalline structures of the prepared MOF particles were studied by XRD. Both the characteristic peaks of the MOF NPs and pMOF MSs were consistent with simulated $\mathrm{NH}_{2}$-UiO-66 (Figure 2d), proving the successful formation of $\mathrm{NH}_{2}$-UiO-66 crystals after solvothermal reaction. ${ }^{54}$ The incorporation of polymer did not disturb the arrangement of frameworks. FTIR spectra of the MOF NPs and pMOF MSs displayed the strong peaks of $\mathrm{O}-\mathrm{Zr}$ at $660 \mathrm{~cm}^{-1},-\mathrm{NH}_{2}$ at 1258, 1652, 3335, and $3460 \mathrm{~cm}^{-1}$, and $\mathrm{O}=\mathrm{C}=\mathrm{O}$ at 1385 and $1566 \mathrm{~cm}^{-1}$, illustrating the homogeneous chemical structures of two MOFs (Figure S1). ${ }^{54-56}$ There was no obvious PSF characteristic peak in the FTIR spectrum of the pMOF MSs owing to the relatively small 
amount of embedded polymers. The peak about $\mathrm{O}=\mathrm{C}=\mathrm{O}$ bond of the pMOF MSs at approximately 1385 $\mathrm{cm}^{-1}$ showed red-shift (Figure 2e). This was ascribed to the dipole-dipole interaction between polar groups of MOFs $(\mathrm{O}=\mathrm{C}=\mathrm{O})$ and PSF (-SO $\left.2_{2}^{-}\right)$, implying the existence of polymer chains in frameworks. To further confirm the polymer embedding, XPS was employed to study the chemical bonding states of the MOF NPs and pMOF MSs (Figure $2 \mathrm{f}$ and Figure S2). High resolution XPS spectra of both MOF NPs and pMOF MSs displayed Zr 3d peaks, while the Zr atomic content decreased from $5.4 \%$ (MOF NPs) to $4.7 \%$ (pMOF MSs ) as no Zr element in PSF. The new S 2p peak of the pMOF MSs proved the successful PSF embedding as well. Based on $0.5 \%$ sulfur atomic content and the molecular formulas of $\mathrm{NH}_{2}$-UiO-66 cells $\left(\mathrm{Zr}_{24} \mathrm{O}_{120} \mathrm{C}_{192} \mathrm{~N}_{24}\right)$ and PSF repeat units $\left(\mathrm{C}_{27} \mathrm{O}_{4} \mathrm{~S}\right)$, the PSF content of the pMOF surface could be roughly calculated at 2.0 units per cell.

The $\mathrm{N}_{2}$ adsorption-desorption isotherms of the MOF NPs and pMOF MSs were measured to investigate the influence of polymer embedding on the porosities of MOFs. Both MOF NPs and pMOF MSs exhibited type-I adsorption behavior (Figure 3a,b), proving their microporous features. The BET surface area of the MOF NPs was calculated to be $725 \mathrm{~m}^{2} \mathrm{~g}^{-1}$, which agreed with that of the $\mathrm{NH}_{2}$-UiO-66 particles reported in previous studies. ${ }^{57}$ For the pMOF MSs, the measured BET surface area $\left(799 \mathrm{~m}^{2} \mathrm{~g}^{-1}\right)$ was slight larger than that of the MOF NPs (Table S1). The micropore size distributions of MOF NPs and pMOF MSs were calculated by Non-Local Density Functional Theory (NLDFT) and displayed in Figure 3a,b with two main peaks at 1.1 and $1.3 \mathrm{~nm}$, which were in accordance with those of the typical $\mathrm{NH}_{2}$-UiO-66 particles prepared by solvothermal synthesis. ${ }^{58}$ Compared with the MOF NPs, the larger and smaller pores of the pMOF MSs showed lower and higher peak intensities, respectively, revealing that the PSF chains facilitated the regular arrangements of crystals. The $\mathrm{CO}_{2}, \mathrm{CH}_{4}$, and $\mathrm{N}_{2}$ gas adsorption properties of the MOF NPs and pMOF MSs were measured at $25^{\circ} \mathrm{C}$ (Figure 3c,d). The adsorption capacities of two MOF materials were ordered by the polarizabilities of $\mathrm{CO}_{2}\left(26.3 \times 10^{-25} \mathrm{~cm}^{-3}\right), \mathrm{CH}_{4}\left(26.0 \times 10^{-25} \mathrm{~cm}^{-3}\right)$, and $\mathrm{N}_{2}\left(17.6 \times 10^{-25} \mathrm{~cm}^{-3}\right)$. The pMOF MSs showed slightly higher $\mathrm{CO}_{2}$ uptake of $36.7 \mathrm{~mL} \mathrm{~g}^{-1}$ in contrast with the MOF NPs $\left(32.0 \mathrm{~mL} \mathrm{~g}^{-1}\right)$ due to the larger BET surface area. For $\mathrm{CH}_{4}$ or $\mathrm{N}_{2}$, the MOF NPs and pMOF MSs exhibited similar adsorption capacities. The adsorption selectivity of the prepared MOF NPs and pMOF MSs was calculated by Henry's law (Figure S3). After polymer embedding, the $\mathrm{CO}_{2} / \mathrm{CH}_{4}$ and $\mathrm{CO}_{2} / \mathrm{N}_{2}$ selectivities increased from 4.0 and 13.5 (MOF NPs) to 4.4 and 17.9 (pMOF MSs). 

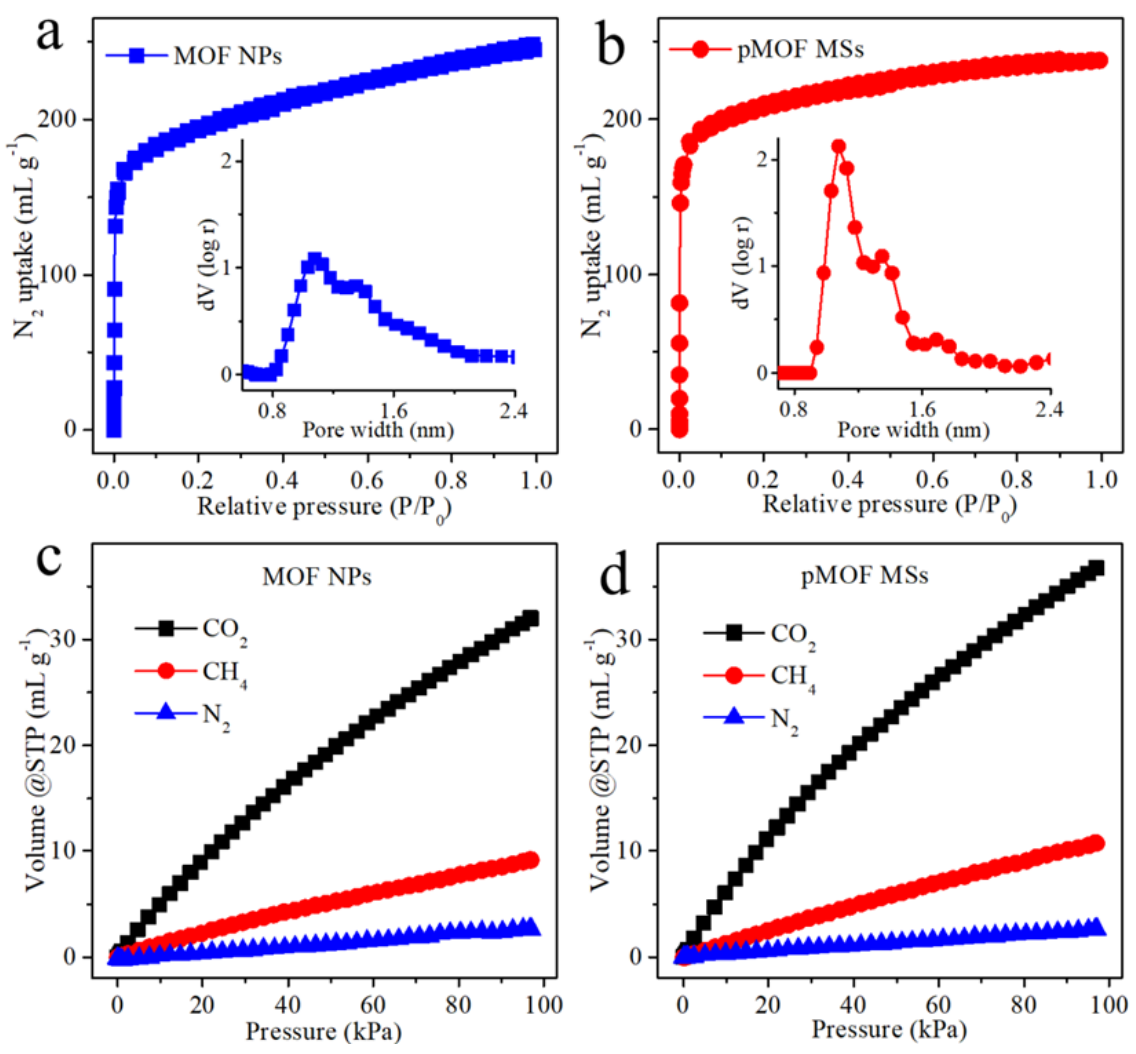

Figure 3. $\mathrm{N}_{2}$ adsorption-desorption isotherms and (inset) pore size distributions of the (a) MOF NPs and (b) pMOF MSs. $\mathrm{CO}_{2}, \mathrm{CH}_{4}$, and $\mathrm{N}_{2}$ adsorption isotherms of the (c) MOF NPs and (d) pMOF MSs.

In order to show the universality of polymer embedding strategy, UiO- $66,{ }^{47}$ constructed by $\mathrm{Zr}_{6} \mathrm{O}_{4}(\mathrm{OH})_{4}$ nodes and 1,4-dicarboxybenzene (BDC) linkers, was also applied in preparing pMOF MSs. The solvothermally synthesized UiO-66 crystals possessed octahedral structure, with particle sizes in the range of 150-200 nm (Figure S4a). Similar to the pMOF MSs, the pUiO-66 MSs prepared by one-pot synthesis also displayed polycrystalline spherical structures with rough surface and diameters of 1-2 $\mu \mathrm{m}$ (Figure S4b,c). The XRD patterns further verified the formation of UiO-66 crystals in pUiO-66 MSs (Figure S4d). The one-pot synthesis for preparation of polymer-embedded MOF MSs was versatile for various MOF materials.

\section{Preparation of MMMs}

After successful synthesis of MOF materials, the MOF NPs and pMOF MSs were mixed in PSF matrixes to fabricate MMMs. The MOF particles and PSF powders were first dispersed and blended in $\mathrm{CHCl}_{3}$ for obtaining homogeneous inks, followed by casting and complete drying for membrane formation. The SEM images of the pristine PSF membrane, MOF-NP/PSF MMM ( $\mathrm{NH}_{2}$-UiO-66 NPs and PSF), and pMOF-MS/PSF MMM (polymer-embedded $\mathrm{NH}_{2}$-UiO-66 MSs and PSF) were captured for observing the microstructures (Figure 4 and Figure S5,S6). Compared with the smooth PSF membrane, the MOF-NP/PSF and pMOF-MS/PSF MMMs displayed rough cross section due to the incorporation of MOF fillers. It could be found that the serious aggregation of fillers and the distinct interfacial defect between two phases appeared in the MOFNP/PSF MMM (Figure 4a,b), which would serve as non-selective pathways for molecular transports and then reduce selectivity. The filler aggregation and the interfacial defect of the pMOF-MS/PSF MMM were alleviated substantially, derived from the identical characteristics of the embedded PSF with the matrix of MMMs as well as the micrometer-sized structures of the pMOF MSs (Figure 4c,d). The broken pMOF MSs and the well cohesive interface suggested that the interfacial binding force was even stronger than that of 
the intrinsic MSs themselves (Figure 4d). The tight interfacial combination confirmed the excellent affinity of the polymeric matrixes to the pMOF MSs.
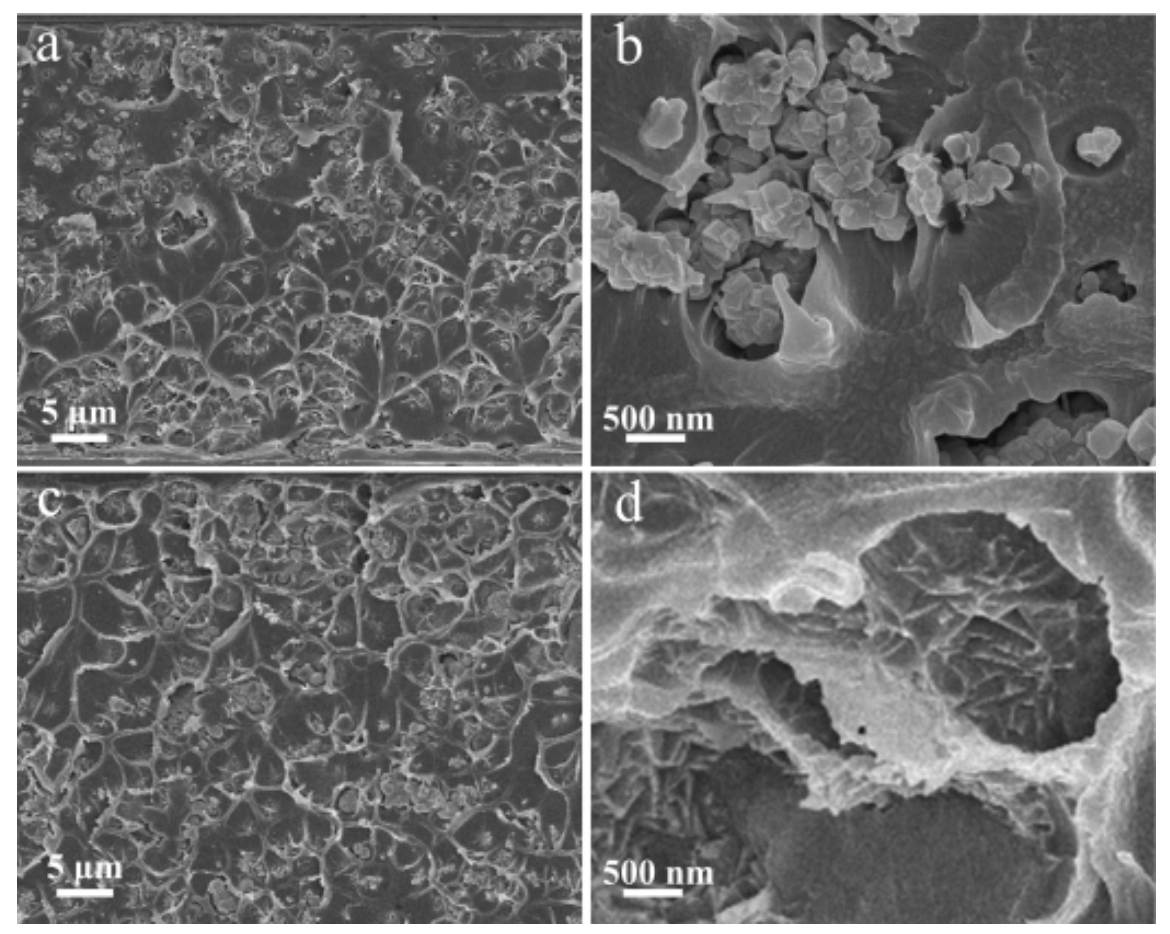

Figure 4. Cross sectional SEM images of the (a,b) MOF-NP/PSF and (c,d) pMOF-MS/PSF MMMs.

\section{Gas separation performance}

To evaluate the separation performance, the gas permeation properties of the prepared membranes was tested and presented in Figure 5a. The pristine PSF membrane exhibited normal $\mathrm{CO}_{2}$ permeability of 5.1 Barrer, in comparison with the membranes reported in previous studies. ${ }^{59,60}$ The $\mathrm{CO}_{2}$ permeability of the MOF-NP/PSF MMM increased by $78 \%$. The enhancement of permeability was attributed to that the large permanent pores, high internal surface area, and invalid defects from filler incorporation of the MOF NPs led to faster transport of $\mathrm{CO}_{2}$ molecules. Since the larger porous MSs provided straighter and longer channels for gas permeation, the pMOF-MS/PSF MMM had even higher permeability of $220 \%$ as the PSF membrane. Analogously, the pUiO-66-MS/PSF MMM displayed large $\mathrm{CO}_{2}$ permeability of $190 \%$ as the PSF membrane. Owing to the aggregation of MOF NPs and the existence of invalid defects, the MOF-NP/PSF MMM exhibited degraded $\mathrm{CO}_{2} / \mathrm{CH}_{4}$ selectivity of 18.0, relative to the pristine PSF membrane with typical selectivity of 20.0. Attractively, the $\mathrm{CO}_{2} / \mathrm{CH}_{4}$ selectivity of the pMOF-MS/PSF MMM reached at 26.1 due to the outstanding compatibility. The pUiO-66-MS/PSF MMM also displayed larger $\mathrm{CO}_{2} / \mathrm{CH}_{4}$ selectivity of 23.6. For $\mathrm{CO}_{2} / \mathrm{N}_{2}$ separation, the incorporation of the pMOF MSs could improve the $\mathrm{CO}_{2} / \mathrm{N}_{2}$ selectivity from 10.9 (PSF membrane) to 16.2 (pMOF-MS/PSF MMM). Compared with the reported MMMs, although the separation performance of the pMOF-MS/PSF MMM was moderate (Table S2), the permeability (2.2) and selectivity (1.3) ratios of MMMs to pristine membranes caused by incorporation of pMOF MSs were impressive (Figure S7 and Table S3). These results strongly demonstrated that the presence of pMOF MSs could not only remarkably accelerate the passport of $\mathrm{CO}_{2}$ molecules through the membranes but also improve the $\mathrm{CO}_{2}$ separation ability due to the reduced interfacial voids and filler aggregations.

\section{Separation mechanism}

For further clarifying the effect of polymer embedding on separation performance, the solubility and dif- 
fusivity of gases through the membranes were simulated (Figure 5b-d and Table S4). As expected, the $\mathrm{CO}_{2}$ adsorption capacity of the MOF-NP/PSF, pMOF-MS/PSF, and pUiO-66-MS/PSF MMMs were much higher than that of the PSF membrane owing to the specific interaction of $\mathrm{Zr}_{6} \mathrm{O}_{4}(\mathrm{OH})_{4}$ clusters and $-\mathrm{NH}_{2}$ groups in MOFs to $\mathrm{CO}_{2}$ molecules. However, because the incorporated MOF fillers simultaneously enhanced the $\mathrm{CO}_{2}$ and $\mathrm{CH}_{4}$ solubilities, the $\mathrm{CO}_{2} / \mathrm{CH}_{4}$ solubility selectivity reduced from 5.3 to about 3.2. The $\mathrm{CO}_{2}$ diffusivity of the MMMs was enhanced after MOF incorporation (Figure 5d), while the $\mathrm{CH}_{4}$ diffusivity of the MMMs declined. This might be explained by that the special interaction between the polymer chains and MOFs narrowed the gas transport channels. ${ }^{42}$ Therefore, the $\mathrm{CO}_{2} / \mathrm{CH}_{4}$ diffusivity selectivity increased drastically. In particular, for the MMMs with polymer-embedded MOF MSs, the $\mathrm{CO}_{2} / \mathrm{CH}_{4}$ diffusivity selectivity of the pMOF-MS/PSF and pUiO-66-MS/PSF MMMs were 9.2 and 8.9, respectively, which were much higher than that of the MOF-NP/PSF MMM (5.7) and pristine PSF membrane (3.8). Therefore, it could be deduced that the great improvement in diffusivity selectivity was the dominate factor for the higher $\mathrm{CO}_{2} / \mathrm{CH}_{4}$ selectivity of the pMOF-MS/PSF and pUiO-66-MS/PSF MMMs. For the MOF-NP/PSF MMMs, the serious filler aggregations and obvious interfacial defects offered the invalid channels for non-selective gas transports. For the pMOF-MS/PSF MMMs, the excellent interfacial compatibility, highly efficient transport channels, and large adsorption capacities contributed to the greater $\mathrm{CO}_{2}$ selectivity and permeability.
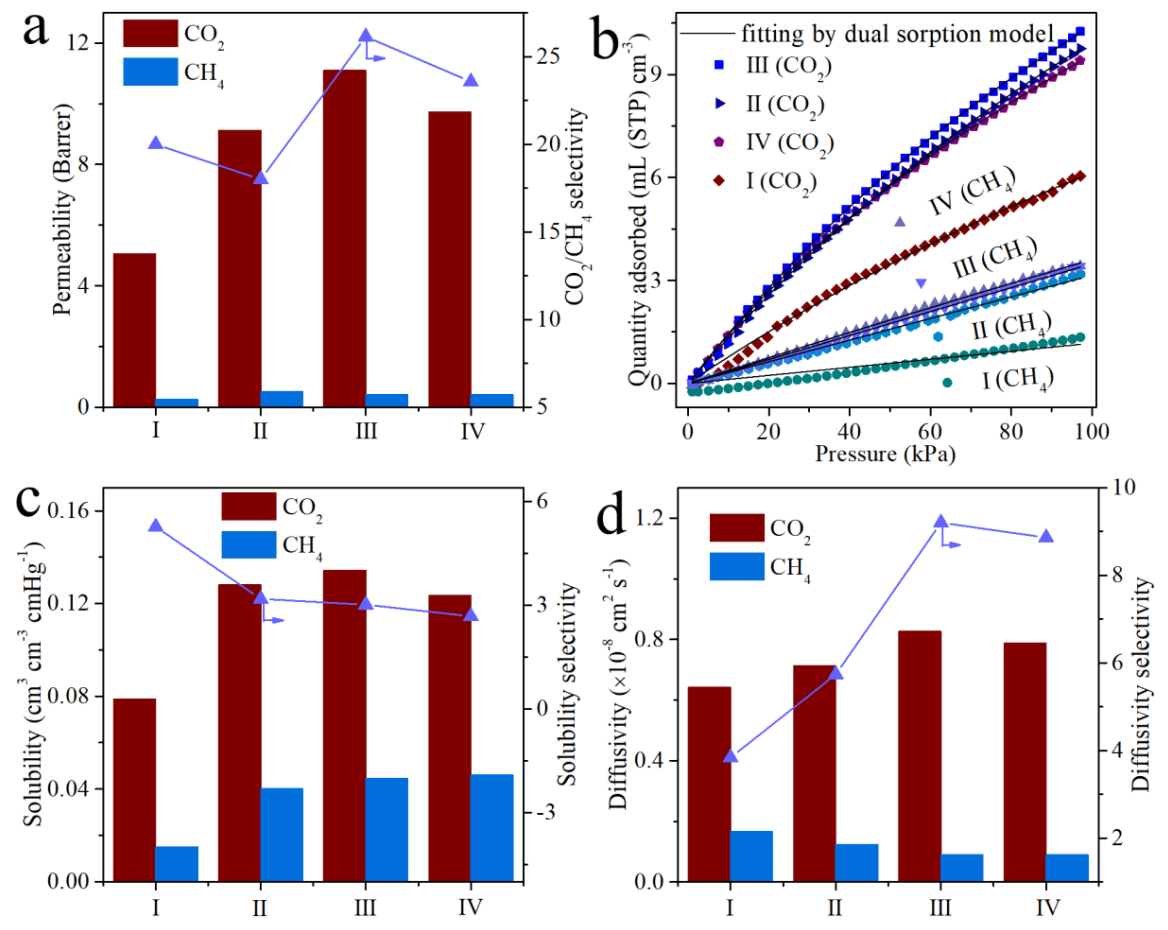

Figure 5. (a) Gas permeation properties and $\mathrm{CO}_{2} / \mathrm{CH}_{4}$ selectivities of the (I) pristine PSF membrane, (II) MOF-NP/PSF, (III) pMOF-MS/PSF, and (IV) pUiO-66-MS/PSF MMMs. (b) $\mathrm{CO}_{2}$ and $\mathrm{CH}_{4}$ adsorption isotherms with the corresponding fitting lines by dual sorption model. (c) Solubility and $\mathrm{CO}_{2} / \mathrm{CH}_{4}$ solubility selectivity of different membranes. (d) Diffusivity and $\mathrm{CO}_{2} / \mathrm{CH}_{4}$ diffusivity selectivity of different membranes.

\section{CONCLUSIONS}

In summary, we have developed a concept of embedding polymer in MOFs by simple one-pot synthesis to improve the separation performance of MMMs. The versatile polymer embedding strategy can promote the formation of large MOF microspheres and strengthen the interaction of fillers to polymer matrixes, while maintaining the crystalline structures of MOFs. Thanks to the elimination of the interfacial voids and filler 
aggregations from embedding polymer, and the formation of superior transport channels from incorporating large pMOF MSs, the prepared MMMs with excellent compatibility exhibit greatly enhanced selectivity and permeability. For examples, the pMOF-MS/PSF MMMs show $220 \%$ and $123 \% \mathrm{CO}_{2}$ permeability and $131 \%$ and $145 \% \mathrm{CO}_{2} / \mathrm{CH}_{4}$ selectivity as the pure PSF membranes and MOF MMMs, respectively. The substantially increased permeability and selectivity, coupled with the simple, versatile, and efficient preparation processes, indicate that the polymer-embedding of MOF fillers reported here offers an alternative route to improve filler geometry, interfacial compatibility, and transport passages for obtaining high-performance MMMs.

\section{ACKNOWLEDGMENTS}

This work was financially supported by the National Natural Science Foundation of China (Grant No. 51708252) and the Hong Kong Scholars Program (Grant No. XJ2019046).

\section{REFERENCES}

1. Seoane B, Coronas J, Gascon I, Etxeberria Benavides M, Karvan O, Caro J, Kapteijn F, Gascon J. Metal-organic framework based mixed matrix membranes: a solution for highly efficient $\mathrm{CO}_{2}$ capture? Chem Soc Rev . 2015;44:2421-2454.

2. Miller DC, Litynski JT, Brickett LA, Morreale BD. Toward transformational carbon capture systems. AIChE J . 2016;62:2-10.

3. Sinha A, Realff MJ. A parametric study of the techno-economics of direct $\mathrm{CO}_{2}$ air capture systems using solid adsorbents. AIChE J . 2019;65:e16607.

4. Yang Z, Khan TS, Alshehhi M, AlWahedi YF. Economic assessment of carbon capture by minichannel absorbers. AIChE $J$. 2018;64:620-631.

5. Xian S, Xu F, Zhao Z, Li Y, Li Z, Xia Q, Xiao J, Wang H. A novel carbonized polydopamine (C-PDA) adsorbent with high $\mathrm{CO}_{2}$ adsorption capacity and water vapor resistance.AIChE J. 2016;62:3730-3738.

6. Yu H, Tan Z, Thé J, Feng X, Croiset E, Anderson WA. Kinetics of the absorption of carbon dioxide into aqueous ammonia solutions. AIChE J. 2016;62:3673-3684.

7. Khalilpour R, Abbas A, Lai Z, Pinnau I. Modeling and parametric analysis of hollow fiber membrane system for carbon capture from multicomponent flue gas. AIChE J. 2012;58:1550-1561.

8. Qiao Z. Sheng M, Wang J, Zhao S, Wang Z. Metal-induced polymer framework membrane with high performance for $\mathrm{CO}_{2}$ separation. AIChE J. 2019;65:239-249.

9. Li W. Zhang G, Zhang C, Meng Q, Fan Z, Gao C. Synthesis of trinity metal-organic framework membranes for $\mathrm{CO}_{2}$ capture. Chem Commun . 2014;50:3214-3216.

10. Lima FV, Daoutidis P, Tsapatsis M. Modeling, optimization, and cost analysis of an IGCC plant with a membrane reactor for carbon capture. AIChE J . 2016;62:1568-1580.

11. Cai L, Wu X, Zhu X, Ghoniem AF, Yang W. High-performance oxygen transport membrane reactors integrated with IGCC for carbon capture.AIChE J . 2020;66:e16427.

12. Robeson LM. The upper bound revisited. J Membr Sci . 2008;320:390-400.

13. Park HB, Kamcev J, Robeson LM, Elimelech M, Freeman BD. Maximizing the right stuff: the trade-off between membrane permeability and selectivity. Science . 2017;356:eaab0530.

14. Dechnik J, Gascon J, Doonan CJ, Janiak C, Sumby CJ. Mixed-matrix membranes. Angew Chem Int Ed . 2017;56:9292-9310.

15. Bachman JE, Smith ZP, Li T, Xu T, Long JR. Enhanced ethylene separation and plasticization resistance in polymer membranes incorporating metal-organic framework nanocrystals. Nat Mater . 2016;15:845-849.

16. Shen J, Zhang M, Liu G, Guan K, Jin W. Size effects of graphene oxide on mixed matrix membranes for $\mathrm{CO}_{2}$ separation. AIChE J . 2016;62:2843-2852.

17. Zhang J, Schott JA, Li Y, Zhan W, Mahurin SM, Nelson K, Sun XG, Paranthaman MP, Dai S. Membrane-based gas separation accelerated by hollow nanosphere architectures. Adv Mater . 2017;29:1603797.

18. Ghalei B, Sakurai K, Kinoshita Y, Wakimoto K, Isfahani AP, Song QL, Doitomi K, Furukawa S, 
Hirao H, Kusuda H, Kitagawa S, Sivaniah E. Enhanced selectivity in mixed matrix membranes for $\mathrm{CO}_{2}$ capture through efficient dispersion of amine-functionalized MOF nanoparticles. Nat Energy . 2017;2:17086.

19. Zhang W, Liu D, Guo X, Huang H, Zhong C. Fabrication of mixed-matrix membranes with MOFderived porous carbon for $\mathrm{CO}_{2}$ separation. AIChE J . 2018;64:3400-3408.

20. Qiu S, Xue M, Zhu G. Metal-organic framework membranes: from synthesis to separation application. Chem Soc Rev . 2014;43:6116-6140.

21. Li W, Zhang Y, Li Q, Zhang G. Metal-organic framework composite membranes: synthesis and separation applications. Chem Eng Sci . 2015;135:232-257.

22. Rui Z, James JB, Kasik A, Lin YS. Metal-organic framework membrane process for high purity $\mathrm{CO}_{2}$ production. AIChE J . 2016;62:3836-3841.

23. Liu Y, Ban Y, Yang W. Microstructural engineering and architectural design of metal-organic framework membranes. Adv Mater . 2017;29:1606949.

24. Li W. Metal-organic framework membranes: production, modification, and applications. Prog Mater Sci . 2019;100:21-63.

25. Wu W, Li Z, Chen Y, Li W. Polydopamine-modified metal-organic framework membrane with enhanced selectivity for carbon capture.Environ Sci Technol . 2019;53:3764-3772.

26. Hou J, Hong X, Zhou S, Wei Y, Wang H. Solvent-free route for metal-organic framework membranes growth aiming for efficient gas separation. AIChE $J$. 2019;65:712-722.

27. Shen J, Liu G, Huang K, Li Q, Guan K, Li Y, Jin W. UiO-66-polyether block amide mixed matrix membranes for $\mathrm{CO}_{2}$ separation.J Membr Sci . 2016;513:155-165.

28. Marti AM, Wickramanayake W, Dahe G, Sekizkardes A, Bank TL, Hopkinson DP, Venna SR. Continuous flow processing of ZIF-8 membranes on polymeric porous hollow fiber supports for $\mathrm{CO}_{2}$ capture. ACS Appl Mater Interfaces . 2017;9:5678-5682.

29. Rodenas T, Luz I, Prieto G, Seoane B, Miro H, Corma A, Kapteijn F, Xamena FXL, Gascon J. Metal-organic framework nanosheets in polymer composite materials for gas separation. Nat Mater . 2015;14:48-55.

30. Wu X, Ren Y, Sui G, Wang G, Xu G, Yang L, Wu Y, He G, Nasir N, Wu H, Jiang Z. Accelerating $\mathrm{CO}_{2}$ capture of highly permeable polymer through incorporating highly selective hollow zeolite imidazolate framework. AIChE J . 2020;66:e16800.

31. Lin R, Hernandez BV, Ge L, Zhu Z. Metal organic framework based mixed matrix membranes: an overview on filler/polymer interfaces. J Mater Chem A . 2018;6:293-312.

32. Zhang C, Dai Y, Johnson JR, Karvan O, Koros WJ. High performance ZIF-8/6FDA-DAM mixed matrix membrane for propylene/propane separations. J Membr Sci . 2012;389:34-42.

33. Rodenas T, van Dalen M, Garcia-Perez E, Serra-Crespo P, Zornoza B, Kapteijn F, Gascon J. Visualizing MOF mixed matrix membranes at the nanoscale: towards structure-performance relationships in $\mathrm{CO}_{2} / \mathrm{CH}_{4}$ separation over $\mathrm{NH}_{2}$-MIL-53(Al)@PI. Adv Funct Mater . 2014;24:249-256.

34. Japip S, Xiao Y, Chung TS. Particle size effects on gas transport properties of 6FDA-Durene/ZIF-71 mixed matrix membranes. Ind Eng Chem Res . 2016;55:9507-9517.

35. Ma X, Wu X, Caro J, Huang A. Polymer composite membrane with penetrating ZIF-7 sheets displays high hydrogen permselectivity. Angew Chem Int Ed . 2019;58:16156-16160.

36. Sanchez-Lainez J, Zornoza B, Friebe S, Caro J, Cao S, Sabetghadam A, Seoane B, Gascon J, Kapteijn F, Guillouzer CL, Clet G, Daturi M, Tellez C, Coronas J. Influence of ZIF-8 particle size in the performance of polybenzimidazole mixed matrix membranes for pre-combustion $\mathrm{CO}_{2}$ capture and its validation through interlaboratory test. J Membr Sci . 2016;515:45-53.

37. Denny MS, Moreton JC, Benz L, Cohen SM. Metal-organic frameworks for membrane-based separations. Nat Rev Mater. 2016;1:16078.

38. Venna SR, Lartey M, Li T, Spore A, Kumar S, Nulwala HB, Luebke DR, Rosi NL, Albenze E. Fabrication of MMMs with improved gas separation properties using externally-functionalized MOF particles. J Mater Chem A . 2015;3:5014-5022.

39. Wang Z, Wang D, Zhang S, Hu L, Jin J. Interfacial design of mixed matrix membranes for improved 
gas separation performance. Adv Mater . 2016;28:3399-3405.

40. Lin R, Ge L, Diao H, Rudolph V, Zhu Z. Ionic liquids as the MOFs/polymer interfacial binder for efficient membrane separation. ACS Appl Mater Interfaces . 2016;8:32041-32049.

41. Molavi H, Shojaei A, Mousavi SA. Improving mixed-matrix membrane performance via PMMA grafting from functionalized $\mathrm{NH}_{2}$-UiO-66. J Mater Chem A . 2018;6:2775-2791.

42. Xiang L, Sheng L, Wang C, Zhang L, Pan Y, Li Y. Amino-functionalized ZIF-7 nanocrystals: improved intrinsic separation ability and interfacial compatibility in mixed-matrix membranes for $\mathrm{CO}_{2} / \mathrm{CH}_{4}$ separation. Adv Mater. 2017;29:1606999.

43. Qian Q, Wu AX, Chi WS, Asinger PA, Lin S, Hypsher A, Smith ZP. Mixed-matrix membranes formed from imide-functionalized UiO-66- $\mathrm{NH}_{2}$ for improved interfacial compatibility.ACS Appl Mater Interfaces . 2019;11:31257-31269.

44. Sun D, Li Z. Double-solvent method to Pd nanoclusters encapsulated inside the cavity of $\mathrm{NH}_{2}$-Uio66(Zr) for efficient visible-light-promoted suzuki coupling reaction. J Phys Chem C . 2016;120:1974419750 .

45. Vieth WR, Howell JM, Hsieh JH. Dual sorption theory. J Membr Sci . 1976;1:177-220.

46. Scholes CA, Stevens GW, Kentish SE. Permeation through $\mathrm{CO}_{2}$ selective glassy polymeric membranes in the presence of hydrogen sulfide. AIChE J. 2012;58:967-973.

47. Cavka JH, Jakobsen S, Olsbye U, Guillou N, Lamberti C, Bordiga S, Lillerud KP. A new zirconium inorganic building brick forming metal organic frameworks with exceptional stability. J Am Chem Soc . 2008;130:13850-13851.

48. Guo X, Liu D, Han T, Huang H, Yang Q, Zhong C. Preparation of thin film nanocomposite membranes with surface modified MOF for high flux organic solvent nanofiltration. AIChE J. 2017;63:1303-1312.

49. Chun J, Kang S, Park N, Park EJ, Jin X, Kim KD, Seo HO, Lee SM, Kim HJ, Kwon WH, Park YK, Kim JM, Kim YD, Son SU. Metal-organic framework@microporous organic network: hydrophobic adsorbents with a crystalline inner porosity. J Am Chem Soc . 2014;136:6786-6789.

50. Guo X, Huang H, Ban Y, Yang Q, Xiao Y, Li Y, Yan W, Zhong C. Mixed matrix membranes incorporated with amine-functionalized titanium-based metal-organic framework for $\mathrm{CO}_{2} / \mathrm{CH}_{4}$ separation. J Membr Sci . 2015;478:130-139.

51. Ban Y, Li Z, Li Y, Peng Y, Jin H, Jiao W, Guo A, Wang P, Yang Q, Zhong C, Yang W. Confinement of ionic liquids in nanocages: tailoring the molecular sieving properties of ZIF-8 for membrane-based $\mathrm{CO}_{2}$ capture. Angew Chem Int Ed . 2015;54:15483-15487.

52. Li H, Meng F, Zhang S, Wang L, Li M, Ma L, Zhang W, Zhang W, Yang Z, Wu T, Lee S, Huo F, Lu J. Crystal-growth-dominated fabrication of metal-organic frameworks with orderly distributed hierarchical porosity. Angew Chem Int Ed . 2020;59:2457-2464.

53. Zhang Z, Nguyen HTH, Miller SA, Cohen SM. PolyMOFs: a class of interconvertible polymer-metalorganic-framework hybrid materials. Angew Chem Int Ed . 2015;54:6152-6157.

54. Tien-Binh N, Rodrigue D, Kaliaguine S. In-situ cross interface linking of PIM-1 polymer and UiO-66$\mathrm{NH}_{2}$ for outstanding gas separation and physical aging control. J Membr Sci . 2018;548:429-438.

55. Jiang Y, Liu C, Caro J, Huang A. A new UiO-66- $\mathrm{NH}_{2}$ based mixed-matrix membranes with high $\mathrm{CO}_{2} / \mathrm{CH}_{4}$ separation performance.Micropor Mesopor Mat . 2019;274:203-211.

56. Molavi H, Eskandari A, Shojaei A, Mousavi SA. Enhancing $\mathrm{CO}_{2} / \mathrm{N}_{2}$ adsorption selectivity via postsynthetic modification of $\mathrm{NH}_{2}$-UiO-66(Zr). Micropor Mesopor Mat . 2018;257:193-201.

57. Wang Z, Ren H, Zhang S, Zhang F, Jin J. Polymers of intrinsic microporosity/metal-organic framework hybrid membranes with improved interfacial interaction for high-performance $\mathrm{CO}_{2}$ separation. J Mater Chem A . 2017;5:10968-10977.

58. Xiong C, Wang S, Hu P, Huang L, Xue C, Yang Z, Zhou X, Wang Y, Ji H. Efficient selective removal of $\mathrm{Pb}(\mathrm{II})$ by using 6-aminothiouracil-modified Zr-based organic frameworks: from experiments to mechanisms. ACS Appl Mater Interfaces . 2020;12:7162-7178.

59. Kim S, Marand E. Polysulfone and mesoporous molecular sieve MCM-48 mixed matrix membranes for gas separation. Chem Mater . 2006;18:1149-1155.

60. Cheng Y, Ying Y, Zhai L, Liu G, Dong J, Wang Y, Christopher MP, Long S, Wang Y, Zhao D. Mixed 
matrix membranes containing MOF@COF hybrid fillers for efficient $\mathrm{CO}_{2} / \mathrm{CH}_{4}$ separation. $J \mathrm{Membr}$ Sci . 2019;573:97-106. 